\title{
Selection of Reference Genes for qRT-PCR in Bletilla striata under Heat Stress
}

\author{
Fang Liang ${ }^{1}$, Suhua Jiang ${ }^{1}$, Ping'an Hao ${ }^{2}$, Yan Zhang ${ }^{1}$, Shenping $\mathrm{Xu}^{1}$, Suyan Niu ${ }^{1}$, Shiming $\mathrm{Han}^{3}$, Xiuyun Yuan ${ }^{1}$ and \\ Bo Cui ${ }^{*}$ \\ ${ }^{1}$ Bioengineering Research Center, Zhengzhou Normal University, Zhengzhou, Henan Province 450044, China \\ ${ }^{2}$ College of Life Sciences, Northwest Agriculture and Forestry University, Yangling, Shaanxi Province, 712000, China \\ ${ }^{3}$ School of Biological Sciences and Technology, Liupanshui Normal University, Liupanshui, Guizhou Province, 553004, China \\ *For correspondence: cuibo@zznu.edu.cn \\ Received 25 August 2020; Accepted 19 October 2020; Published 25 January 2021
}

\begin{abstract}
Bletilla striata (Thunb.) Reihb.f., a traditional Chinese herbal medicine, has attracted increasing attention because of its wide range of applicability to the medical field and chemical industry. $B$. striata has been identified to be particularly sensitive to high temperatures. Thus, the study of temperature stress on gene transcription is of interest in the field. Use of reliable reference genes is essential for qRT-PCR analysis of genes. However, little information regarding suitable reference genes for the genus Bletilla has been published. In this study, the sequences of seven potential reference genes in $B$. striata were obtained via a homology cloning strategy. These genes were as follows: glyceraldehydes-3-phosphate dehydrogenase $(G A P D H), 18 \mathrm{~S}$ ribosomal RNA $(18 S)$, elongation factor 1 alpha $(E F 1 \alpha)$, $\alpha$-tubulin $(T U A), \beta$-tubulin $(T U B)$, ubiquitin $(U B I)$, and NAC domain protein $(N A C)$. We then evaluated the stability levels of these transcripts in different tissues (root, tuber, and leaf) exposed to high temperature using three conventional software and comprehensive RefFinder algorithms. The results indicated that $18 S$ and $T U B$ were the best internal control genes among different periods of heat treatment and that a combination of $18 S$ and $U B I$ was the best in different tissues. Altogether, $18 S$ and $U B I$ were identified to be the best reference genes for all the samples, while $N A C$ and $T U A$ were the least stable reference genes. The results will be useful for studies on target gene expression in plants of the Bletilla genus. (C) 2021 Friends Science Publishers
\end{abstract}

Keywords: Bletilla; 18S ribosomal RNA; Ubiquitin; Heat stress; Quantitative real-time PCR

\section{Introduction}

Bletilla striata (Thunb.) Reihb.f. is a kind of perennial herbaceous plant belonging to the Bletilla genus of Orchidaceae. The dried tuber is usually regarded as medicinal and is named "Bai-ji" in Chinese. This tuber is considered to be a precious traditional Chinese herbal medicine. Hundreds of compounds have already been isolated from $B$. striata, such as polysaccharides, bibenzyls, stilbenes, phenanthrenes, triterpenoids and steroids (Zhang et al. 2019). These compounds have a variety of biological activities and functions. In traditional medicine, $B$. striata has been widely used for thousands of years to stop bleeding mainly from the stomach and lungs and for detumescence. Modern pharmacology research has further proven that $B$. striata fights against bacteria (Li et al. 2014; Guo et al. 2016; Jiang et al. 2019), influenza A virus (Shi et al. 2017), fibrosis (Wang et al. 2014) and tumorigenesis (Zhan et al. 2014) and promotes wound (Diao et al. 2008; Luo et al. 2010) and oral ulcer healing (Liao et al. 2019). Additionally, $B$. striata is used as a hemostatic agent since it promotes rapid blood coagulation (Hung and Wu 2016; Zhang et al. 2017). "Yunnan Bai Yao", made mainly from B. striata, has been popular for wound healing for more than a hundred years. In addition to applications in the medical field, $B$. striata has also been widely used in food and chemical industries because of its high anti-oxidative (Qu et al. 2016) and anti-aging (Lee et al. 2013) activities. Facial masks containing products derived from $B$. striata have whitening effects and prevent or cure common oral and dental disease when added to toothpaste with negligible adverse effects.

B. striata is also identified as a high-end ornamental flower because of its bright purple perianths and pleasant fragrance. Due to its wide range of application, the demand for $B$. striata has increased sharply. Meanwhile, B. striata possesses the general character of orchids in that its seed has no endosperm. Therefore, the natural reproduction rate is determined to be very low. The increasing demand and low reproduction rate of $B$. striata have resulted in the rapid depletion of $B$. striata as a wild resource. Therefore, artificial or semi-artificial cultivation has been adopted for B. striata in many areas. Currently, B. striata is under 
second-class protection on the national rare and endangered wild plant conversion list in China (Zhang et al. 2019).

B. striata is mainly distributed between 100 and 3200 meters of altitude in south and southwest China, Japan, Thailand, and Myanmar (Zhuang et al. 2019). It grows in damp gullies or hillsides, prefers shade and humid environments, and has no resistance to high temperatures or sun exposure. The leaves turn yellow, and development is inhibited significantly under ambient temperatures higher than $36^{\circ} \mathrm{C}$. Therefore, the discovery of heat-resistant genes is one of the most important efforts in breeding research on B. striata.

Quantitative real-time PCR (qRT-PCR) analysis, with many benefits of simplicity, accuracy, specificity, short turn-around time and high-throughput characteristics, has been used in a variety of fields about study relative expression level of target genes (Bustin et al. 2005; Huggett et al. 2005; Shukla et al. 2019). There are many rules that must be followed to ensure reproducible and accurate results using qRT-PCR (Udvardi et al. 2008; Derveaux et al. 2010). Among them, use of reliable reference genes for data normalization is crucial for proper analysis (Gutierrez et al. 2008). Previous reports have suggested that expression profiles of reference genes vary between species, tissues, and treatments, even that of genes that are widely used as references (Argyropoulos et al. 2006). No single reference gene has been determined that is always expressed stably under any condition (Argyropoulos et al. 2006). Accordingly, it is crucial to discover suitable internal control genes with stable expression for the study of specific transcriptional profiles of genes of interest under a certain experimental condition for a certain species (Yang et al. 2019). There are many reports on screening for proper reference genes in plants, however, there has been no report regarding reference genes for the study of $B$. striata.

In this study, seven potential reference genes were isolated from the leaves of $B$. striata using a homologous cloning method, and then, gene-specific primers for qRTPCR were designed. The transcription levels of these candidates in different tissues with heat treatment for different durations were measured using qRT-PCR, and the stability of the transcript levels was evaluated using three conventional statistical software and comprehensive RefFinder algorithms. Moreover, the expression profile of one target gene which involved in photosynthesis, BsrbcL, was analyzed to verify the reliability of selected reference genes. These results are of significance to the study of genes involved in high temperature resistance and genetic breeding for B. striata.

\section{Materials and Methods}

\section{Plant materials and treatments}

Two-year-old B. striata plants were selected and pre-cultured in a growth chamber (PERCIVAL E-41HO2, USA) under controlled conditions $26^{\circ} \mathrm{C}, 12 \mathrm{~h}$ light/12 h dark, $70 \%$ relative humidity, $100 \mu \mathrm{mol} \cdot \mathrm{m}^{-2} \cdot \mathrm{s}^{-1}$ of light intensity for a week. Then, the plants were cultured at $40^{\circ} \mathrm{C}$ to induce high temperature stress for different durations and the other conditions remained unaltered. Five seedlings were used for each sampling, and the experiment was replicated three times. The leaves, tubers, and roots were sampled separately at $0,1,2,4,8,12,24$, and $48 \mathrm{~h}$ under high temperature stress. Then all samples were immediately frozen in liquid nitrogen and stored at $-80^{\circ} \mathrm{C}$ for further step.

\section{Template preparation}

Total RNA from different tissues of $B$. striata seedlings was then extracted using an HP Plant RNA Kit R6837-01 (OMEGA Biotech, China). Then, a RevertAid First Strand cDNA Synthesis Kit was used to synthesize the first cDNA strand for ordinary PCR and clone the candidate reference genes. PrimeScript RT reagent Kit with gDNA Eraser (TakaRa, Japan) was used to synthesize the first cDNA strand for real-time PCR.

\section{Isolation of potential reference genes}

A total of seven genes for candidates including glyceraldehydes-3-phosphate dehydrogenase $(G A P D H), \alpha$ tubulin (TUA), $\beta$-tubulin (TUB), 18S ribosomal RNA (18S), ubiquitin $(U B I)$, elongation factor 1 alpha $(E F 1 \alpha)$, and NAC domain protein $(N A C)$ were selected for expression studies in B. striata under high temperature stress. Degenerate primers were designed according to conserved regions of the seven candidate genes. The reaction conditions for ordinary PCR were as follows: 5 min for pre-denaturing at $94^{\circ} \mathrm{C}$, followed by 35 cycles of denaturing for $40 \mathrm{~s}$ at $94^{\circ} \mathrm{C}$, annealing for $40 \mathrm{~s}$ at $55-58^{\circ} \mathrm{C}$, and extension for $40-80 \mathrm{~s}$ at $72^{\circ} \mathrm{C}$. Additionally, extension at $72^{\circ} \mathrm{C}$ for $10 \mathrm{~min}$ was performed as the final step.

\section{qRT-PCR analysis of candidate genes}

Specific primer pairs for qRT-PCR were designed according to the obtained seven gene sequences isolated from $B$. striata using ordinary PCR. Each reaction mixture contained $10 \mu \mathrm{L}$ of $2 \times$ SYBR Premix Ex Taq II, $2 \mu \mathrm{L}$ of cDNA, $0.8 \mu \mathrm{L}$ of primer $(10 \mu \mathrm{M})$ and add $\mathrm{ddH}_{2} \mathrm{O}$ to the total volume of $20 \mu \mathrm{L}$. Reaction conditions were as follows: $30 \mathrm{~s}$ for pre-denaturing at $95^{\circ} \mathrm{C}$ and 40 cycles of $15 \mathrm{~s}$ at $95^{\circ} \mathrm{C}, 15 \mathrm{~s}$ at $58^{\circ} \mathrm{C}$, and $15 \mathrm{~s}$ at $72^{\circ} \mathrm{C}$. Melting curve analysis was conducted by melting the templates at temperatures from $60^{\circ} \mathrm{C}$ to $95^{\circ} \mathrm{C}$. Amplification efficiencies $(E)$ and correlation coefficients $\left(R^{2}\right)$ of each primer pair for the seven genes were obtained based on serial tenfold dilutions of pooled cDNA.

\section{Detection of candidate reference genes stability}

Transcript level stability of seven genes from $B$. striata in leaves with different periods under high temperature and in different tissues treated for 0 and $8 \mathrm{~h}$ was evaluated using 
three algorithms: geNorm (Vandesompele et al. 2002), NormFinder (Andersen et al. 2004) and BestKeeper (Pfaffl et al. 2004). The optimum reference gene was screened using geNorm software based on calculation of the average transcript level stability value ( $\mathrm{M}$ value) of each candidate. Generally, this software suggests at least two optimum reference genes for transcript level normalization, making the results more accurate. $M$ values $<1.5$ were considered to be acceptable. The lowest $M$ value implied the most stable and vice versa. NormFinder software was used to assay transcript level stability based on intra-class variance and inter-class variance. The ranks and stability values were directly recorded to determine the single most stable gene. BestKeeper algorithm screened out the best gene according to standard deviations (SD) and coefficients of variation (CV) of Ct values. Finally, RefFinder (Xie et al. 2012) was used to integrate and analyze the comprehensive ranking.

\section{Verification of selected reference genes}

To validate the transcript level stability of identified genes, the relative expression level of Bs $r b c L$ gene, which encodes the large subunit of ribulose-1,5-bisphosphate carboxylase/oxygenase (RuBisCO), was measured using qRT-PCR analysis.

\section{Results}

\section{Isolation of candidate genes}

Seven genes were studied for screening of suitable reference genes for analysis of transcript levels of target genes in $B$. striata under high temperature stress. Partial mRNA fragments of the seven candidates were isolated from the leaf of B. striata using homologous cloning. Fragments ranged from $357 \mathrm{bp}$ to $1,792 \mathrm{bp}$ and had $87-99 \%$ homologous sequences compared to other plants.

\section{Specificity and amplification efficiency of primer pairs}

Quantitative RT-PCR primers for the seven genes were designed according to the sequences obtained using PCR amplification, and their specificity was assayed according to the results of gel electrophoresis and melting curve. As shown in Fig. 1A, the results of electrophoresis indicated that there was only one target band obtained by PCR for each gene, and the positions were corresponding to expected size. The melting curves for each primer set showed a single amplification peak indicating stability and specificity (Fig. 1B). $E$ ranged from 90.9 to $110.2 \%$, and $R^{2}$ varied from 0.981 to 0.999 (Table 1 ).

\section{Expression analysis of candidate genes}

Transcript level stability among different samples is an important criterion in selecting a suitable internal reference gene. The transcript level is presented in the form of a cycle threshold value $(\mathrm{Ct})$, which indicates transcript abundance as measured using qRT-PCR analysis. Lower $\mathrm{Ct}$ values indicate higher transcript abundance, and higher values represent lower abundance. Under high temperature stress, the average $\mathrm{Ct}$ values of seven genes ranged from 12.84 to 29.27 among different treatment durations in leaves (Fig. 2A), while they ranged from 12.35 to 28.93 among different tissues (Fig. 2B).

As shown in Fig. 3, 18S gene had the lowest $\mathrm{Ct}$ value of $12.84 \pm 0.36$ (mean $\pm \mathrm{SD}$ ), thus showing the highest expression level among different treatment durations in leaves and $12.35 \pm 0.50$ among different tissues, followed by $G A P D H$ with $21.07 \pm 0.87$ and $20.78 \pm 0.62$ among different treatment durations in leaves and different tissues, respectively. In contrast, the highest values were $29.27 \pm$ 1.30 for TUA among different treatment durations in leaves and $28.93 \pm 1.66$ for $N A C$ among different tissues, followed by $29.12 \pm 1.37$ for $N A C$ and $27.66 \pm 2.49$ for $T U A$ among different treatment time durations in leaves and different tissues, respectively. These values indicated that these genes had the lowest transcript abundance.

\section{Evaluation of transcript level stability of candidates}

geNorm analysis: The results of geNorm analysis are presented in Table 2. The $M$ values of seven genes expressed in leaves treated for different periods at high temperature and in different tissues treated with high temperature for 0 and $8 \mathrm{~h}$ were all determined to be lower than 1.50 with the exception of $T U A$ in different tissues. Interestingly, the most stable genes were $18 S$ and $U B I$, and the least stable was $N A C$ in the two treatment groups. Under different periods of high temperature treatment, $18 S$ and $U B I$ demonstrated the lowest $\mathrm{M}$ value of 0.69 , followed by $G A P D H$ with a value of 0.77 . In different tissues treated with high temperature for 0 and $8 \mathrm{~h}, 18 S$ and $U B I$ presented the lowest $\mathrm{M}$ value of 0.52 , followed by $E F 1 \alpha$ with an $\mathrm{M}$ value of 0.62 .

NormFinder analysis: As shown in Table 3, under different periods of high temperature treatment, TUB had the lowest $\mathrm{M}$ value of 0.263 , indicating that it was the most stable, followed by $G A P D H$ and $18 S$ with $\mathrm{M}$ values of 0.436 and 0.459 , respectively. $N A C$ was ranked last with a value of 0.921 , suggesting that it was the least stable transcript. In different tissues treated with high temperature for 0 and $8 \mathrm{~h}$, the most stable gene was $U B I$ with an $\mathrm{M}$ value of 0.163 , followed by $18 S$ and $G A P D H$ with $\mathrm{M}$ values of 0.214 and 0.314 , respectively. TUA proved to be the most unstable gene with the highest $M$ value of 1.582 .

BestKeeper analysis: The SD values for $N A C$ among different periods of high temperature treatment and for $N A C$ and TUA genes in different tissues treated with high temperature for 0 and $8 \mathrm{~h}$ were found to be greater than 1.0. According to BestKeeper criteria, transcript levels of these genes were unstable. As shown in Table 4, $18 S$ and EF1a were identified as the most stable transcripts among different periods of high temperature treatment; while $18 \mathrm{~S}$ 
Table 1: Characteristics of qRT-PCR for seven genes

\begin{tabular}{|c|c|c|c|c|c|c|}
\hline Gene name & GenBank ID & Primer sequence $\left(5^{\prime}-3^{\prime}\right)$ & Product length & $\operatorname{Tm}\left({ }^{\circ} \mathrm{C}\right)$ & $E(\%)$ & $R^{2}$ \\
\hline \multirow[t]{2}{*}{$\overline{U B I}$} & MT781955 & F: CGCCGATTACAACATCCAGAA & $102 \mathrm{bp}$ & 83 & 90.9 & 0.986 \\
\hline & & R: TTCTTGGGCTTGGTGTATGTC & & & & \\
\hline \multirow{2}{*}{ GAPDH } & MT781952 & F: CAGTCTTTGGCGTCAGGAA & $177 \mathrm{bp}$ & 85.5 & 92.6 & 0.998 \\
\hline & & R: CAACAACAAACATTGGAGCATC & & & & \\
\hline $18 S$ & MT781956 & $\begin{array}{l}\text { F: TTTATGAAAGACGAACCACTGC } \\
\text { R: TCGGCATCGTTTATGGTTG }\end{array}$ & $121 \mathrm{bp}$ & 81.5 & 93.4 & 0.999 \\
\hline TUA & MT781953 & $\begin{array}{l}\text { F: TTTATGAAAGACGAACCACTGC } \\
\text { R: TGAGGCGGTAAGGGATGAA }\end{array}$ & $126 \mathrm{bp}$ & 83.5 & 100.3 & 0.991 \\
\hline$T U B$ & MT781954 & $\begin{array}{l}\text { F: GGAGGGCAATGTGGCAA } \\
\text { R: TAAGCACAGCCCTCGGAAC }\end{array}$ & $172 \mathrm{bp}$ & 85.2 & 93.5 & 0.996 \\
\hline$E F 1 \alpha$ & MK448293 & $\begin{array}{l}\text { F: GCCGTCCTTATTATTGATTCCA } \\
\text { R: GGATCTTATCAGGATTGTAACCA }\end{array}$ & $233 \mathrm{bp}$ & 82.5 & 99.5 & 0.996 \\
\hline$N A C$ & MT781957 & $\begin{array}{l}\text { F: TGGTATTTCTTCACCCCGC } \\
\text { R: TTGCCTTCCAGTAACCCGA }\end{array}$ & $85 \mathrm{bp}$ & 82 & 110.2 & 0.981 \\
\hline
\end{tabular}

Table 2: Rankings of seven genes calculated using geNorm algorithm

\begin{tabular}{|c|c|c|c|c|}
\hline \multirow[t]{2}{*}{ Rank } & \multicolumn{2}{|c|}{ Different periods } & \multicolumn{2}{|c|}{ Different tissues } \\
\hline & Gene & Stability & Gene & Stability \\
\hline 1 & $18 S / U B I$ & 0.69 & $18 S / U B I$ & 0.52 \\
\hline 2 & GAPDH & 0.77 & $E F 1 \alpha$ & 0.62 \\
\hline 3 & EFla & 0.85 & GAPDH & 0.75 \\
\hline 4 & $T U B$ & 0.92 & $T U B$ & 0.86 \\
\hline 5 & TUA & 1.08 & $N A C$ & 1.22 \\
\hline 6 & $N A C$ & 1.21 & $T U A$ & 1.55 \\
\hline
\end{tabular}

Table 3: Rankings of seven genes calculated by NormFinder algorithm

\begin{tabular}{lllll}
\hline Rank & \multicolumn{2}{c}{ Different periods } & \multicolumn{2}{c}{ Different tissues } \\
\cline { 2 - 5 } & Gene & Stability & Gene & Stability \\
\hline 1 & TUB & 0.263 & UBI & 0.163 \\
2 & GAPDH & 0.436 & $18 S$ & 0.214 \\
3 & I8S & 0.459 & GAPDH & 0.314 \\
4 & UBI & 0.478 & TUB & 0.392 \\
5 & EF1 $\alpha$ & 0.596 & EF1 $\alpha$ & 0.448 \\
6 & TUA & 0.889 & NAC & 1.431 \\
7 & NAC & 0.921 & TUA & 1.582 \\
\hline
\end{tabular}

and GAPDH were the most stable genes in different tissues treated with high temperature for 0 and $8 \mathrm{~h}$. Overall, the most stable transcript was $18 \mathrm{~S}$.

RefFinder analysis: The results of geNorm, NormFinder, and BestKeeper were further integrated and analyzed using RefFinder program. The comprehensive ranking of the seven genes generated by RefFinder is shown in Table 5, and the rankings of each gene obtained by the four programs are then presented in Fig. 4. The transcript level of $18 S$ was ranked as the most stable among the different periods of high temperature treatment, followed by $T U B$ and $U B I$. Transcripts of $18 S$ and $U B I$ were suggested to be the most stable in different tissues treated with high temperature for 0 and $8 \mathrm{~h}$. Transcripts of $18 S$ and $U B I$ were ranked as the two highest among all samples.

\section{Verification of the selected reference genes}

In order to verify the utility of the proposed internal control genes, the relative transcript levels of the $B s r b c L$ gene in
Table 4: Rankings of seven genes calculated by BestKeeper algorithm

\begin{tabular}{|c|c|c|c|c|c|c|}
\hline \multirow[t]{2}{*}{ Rank } & \multicolumn{3}{|c|}{ Different periods } & \multicolumn{3}{|c|}{ Different tissues } \\
\hline & Gene & SD & $\mathrm{CV}$ & Gene & SD & $\mathrm{CV}$ \\
\hline 1 & $18 S$ & 0.27 & 2.1 & $18 \mathrm{~S}$ & 0.41 & 3.35 \\
\hline 2 & $E F 1 \alpha$ & 0.60 & 2.39 & GAPDH & 0.48 & 2.31 \\
\hline 3 & $U B I$ & 0.64 & 2.5 & $E F 1 \alpha$ & 0.56 & 2.35 \\
\hline 4 & $T U B$ & 0.66 & 2.56 & $U B I$ & 0.64 & 2.66 \\
\hline 5 & GAPDH & 0.73 & 3.45 & $T U B$ & 0.92 & 3.69 \\
\hline 6 & $T U A$ & 0.91 & 3.1 & $N A C$ & 1.19 & 4.13 \\
\hline 7 & $N A C$ & 1.03 & 3.54 & TUA & 1.89 & 6.83 \\
\hline
\end{tabular}

Table 5: Comprehensive analysis results of seven genes stability obtained by RefFinder program

\begin{tabular}{|c|c|c|c|c|c|c|}
\hline \multirow[t]{2}{*}{ Rank } & \multicolumn{2}{|c|}{ Different periods } & \multicolumn{2}{|c|}{ Different tissues } & \multicolumn{2}{|c|}{ All samples } \\
\hline & Gene & Stability & Gene & Stability & Gene & Stability \\
\hline 1 & $18 \mathrm{~S}$ & 1.86 & $18 S / U B I$ & 1.41 & $18 S$ & 1.57 \\
\hline 2 & $T U B$ & 2.12 & & & $U B I$ & 2.21 \\
\hline 3 & $U B I$ & 2.45 & GAPDH & 2.91 & $E F 1 \alpha / T U B$ & 2.94 \\
\hline 4 & GAPDH & 2.78 & $E F 1 \alpha$ & 3.66 & & \\
\hline 5 & $E F 1 \alpha$ & 3.76 & $T U B$ & 4.73 & GAPDH & 3.36 \\
\hline 6 & TUA & 6.00 & $N A C$ & 6.00 & TUA & 6.24 \\
\hline 7 & $N A C$ & 7.00 & TUA & 7.00 & $N A C$ & 6.74 \\
\hline
\end{tabular}

leaves of $B$. striata under high temperature treatment were measured using the most stable (18S, UBI, and $18 S$ combined with $U B I)$ and least stable (NAC and TUA individually) reference genes as calibrators. The results showed the transcript level of BsrbcL gradually decreased with increasing treatment time. Similar trends were observed when using $18 S$ alone, $U B I$ alone, or $18 S$ combined with $U B I$ to normalize the data (Fig. $5 \mathrm{~A}$ ). It is worth noting that the expression pattern was more uniform when using $18 \mathrm{~S}$ and $U B I$ simultaneously as calibrators than when using each reference gene individually. However, an incorrect expression profile for $B s r b c L$ was exhibited when the least stable reference genes $N A C$ or $T U A$ were used (Fig. 5B).

\section{Discussion}

Bletilla striata tends to grow in a cool, damp, and ventilated environment, and its vegetative period is very short, spanning from April to September. In October, the leaves 


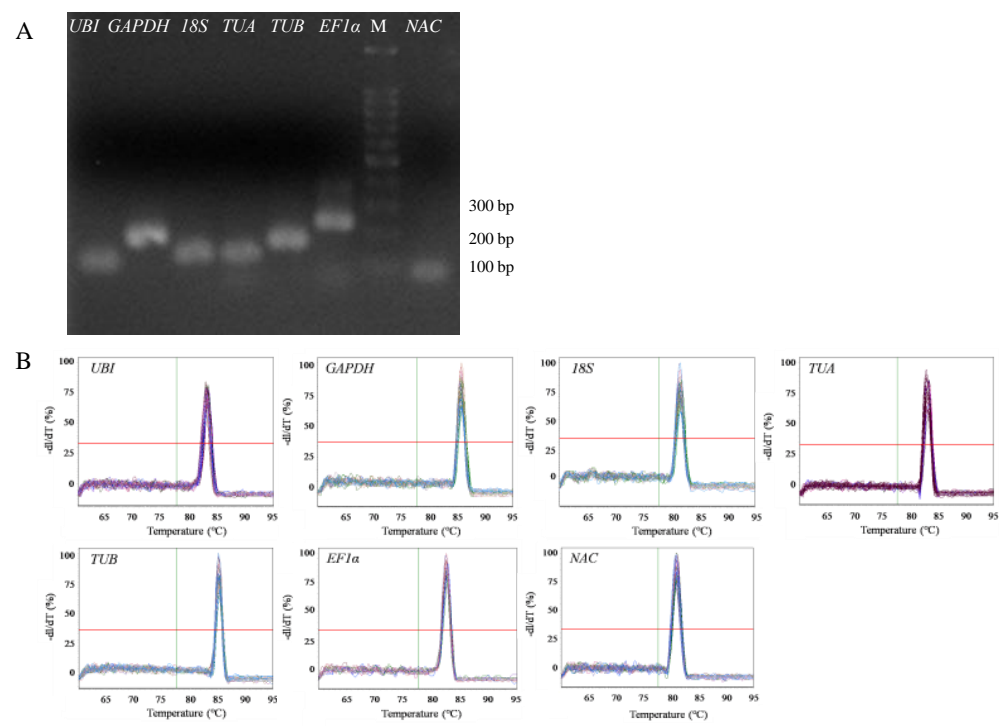

Fig. 1: Specificity of qRT-PCR amplification for the seven genes. (A) PCR products for each gene. (B) Melting curves for qRT-PCR amplification of seven candidate genes

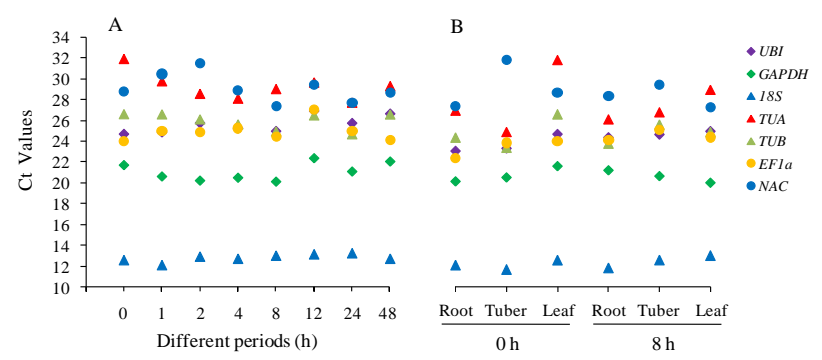

Fig. 2: Ct distributions of seven genes in leaves with different periods of high temperature treatment (A) and in different tissues treated with high temperature for 0 and $8 \mathrm{~h}(\mathrm{~B})$

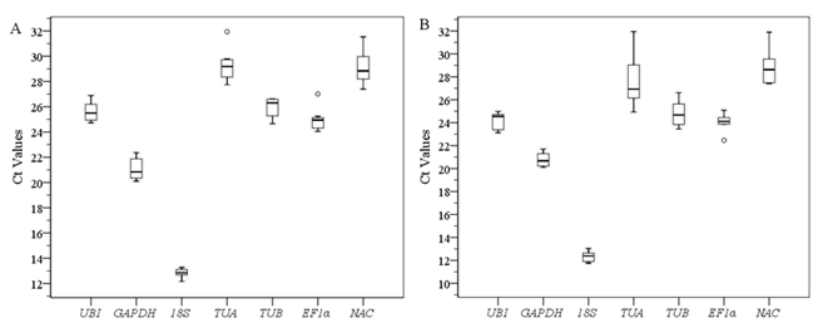

Fig. 3: Ct values of seven candidate genes expressed in leaves with different heat treatment durations (A) and expressed in different tissues treated with high temperature for 0 and $8 \mathrm{~h}(\mathrm{~B})$

start to turn yellow and fall off, at which time the underground stem goes into dormancy. In the central area of China, summer is relatively hot and long. The growth and development of $B$. striata is usually inhibited under high temperature. Therefore, it is essential to study heat-resistant genes and their biological functions in order to breed new heat-resistant varieties of $B$. striata. qRT-PCR is the most commonly used molecular technique for quantifying gene expression level because it has many benefits. The accuracy of quantitative results relies heavily upon suitable internal control genes as normalization factors, which should exhibit stable transcript levels in all samples. Hence, the first step for expression analysis of target genes under a specific experimental condition is the selection of stable internal references. However, little information regarding suitable reference genes for the genus Bletilla has been published.

In this study, the sequences of seven candidate internal control genes were obtained from B. striata, and transcript stabilities of the candidates were evaluated using geNorm, NormFinder, and BestKeeper algorithm. The rankings of seven genes were different for each algorithm. For example, in leaves under different periods of high temperature treatment, $T U B$ was ranked the top which implied the most ideal by NormFinder, while geNorm and BestKeeper identified $18 S$ and $U B I$ as the optimum. Therefore, in the present study, we also used RefFinder to integrate these analyses into a comprehensive ranking of the candidates according to the results of the three algorithms.

In general, internal control genes are typically housekeeping genes, which commonly involves in the processes of basic metabolism and cell components. In the present study, six traditional housekeeping genes including GAPDH, TUA, TUB, $18 S, E F 1 \alpha$, and UBI were selected as candidate reference genes. They have been widely reported and possess good performance within a given species. For example, EFl $\alpha$ was the most appropriate reference gene under cold stress in Eleusine coracana (Jatav et al. 2018), and under drought stress in Setaria italica (Kumar et al. 2013). GAPDH was the best choice in Chinese cabbage (Qi et al. 2010) under drought stress, in Eleusine coracana (Jatav et al. 2018) under cold, salinity or heat stress conditions, and under ABA stress in Polygonum cuspidatum 


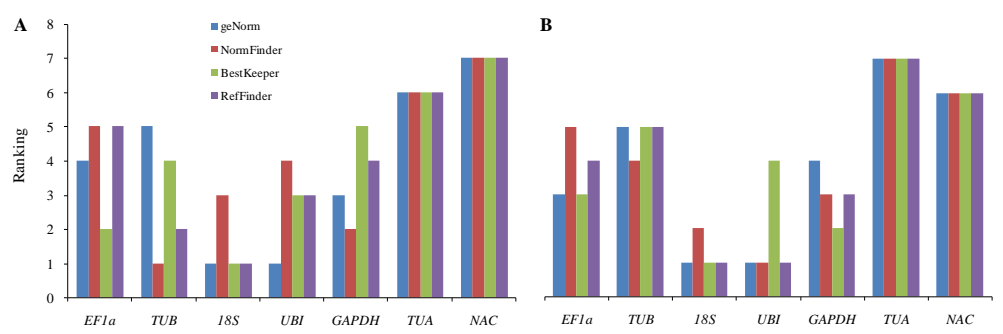

Fig. 4: Comparison of the ranking for each gene based on their M values generated by geNorm, NormFinder, BestKeeper, and RefFinder
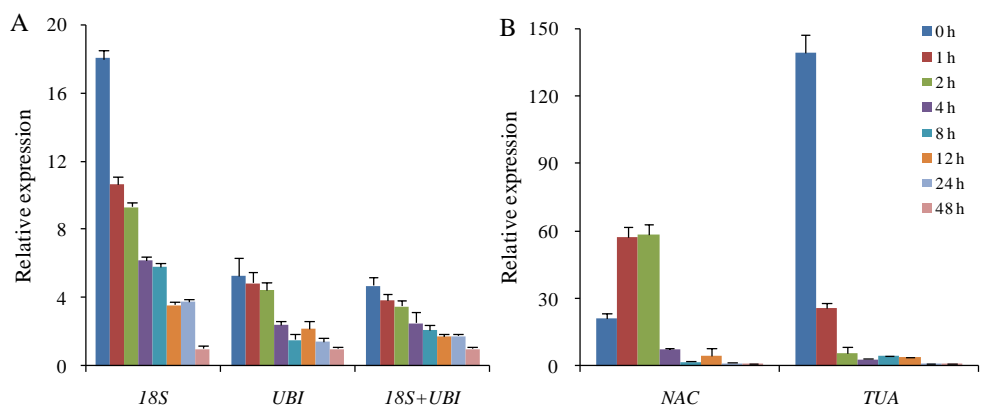

Fig. 5: Relative expression levels of BsrbcL normalized using the selected most stable (A) and unstable genes (B) in leaves of Bletilla striata under high temperature stress

(Wang et al. 2019). UBI showed peak stability under ABA stress in Hordeum brevisubulatum (Zhang et al. 2018), under cold stress in Morus alba (Shukla et al. 2019), under drought stress in wheat (Kiarash et al. 2018; Dudziak et al. 2020), and across different tissues in Miscanthus lutarioriparia (Cheng et al. 2019). The most proper reference gene for cold stress in Hordeum brevisubulatum (Zhang et al. 2018), salinity stress in Panicum virgatum (Huang et al. 2014) and drought stress in Miscanthus sinensis (Zhong et al. 2020) was 18S. TUA was reported as the first stable in different tissues of Hordeum brevisubulatum (Zhang et al. 2018) and PEG-treated stems and leaves of Betula luminifera (Wu et al. 2017). TUB had the highest ranking under drought and cold in Panicum virgatum (Huang et al. 2014), under ABA stress in Polygonum cuspidatum (Wang et al. 2019) and in vegetative tissues of Phalaenopsis (Yuan et al. 2014). Additionally, a novel candidate, NAC domain protein gene, has been used as a candidate reference gene (Lin et al. 2014; Huang et al. 2014). It was reported that NAC was the most stable in stressed roots from Codonopsis pilosula (Cao et al. 2017). Therefore, $N A C$ was added as a candidate gene.

In leaves under different periods of high temperature treatment, $18 S$ and $U B I$ exhibited the most stable transcripts using geNorm, while $T U B$ emerged as the optimal transcript from NormFinder analysis, and $18 S$ was the best candidate as analyzed using BestKeeper and RefFinder. However, the results obtained by the four methods were consistent in that the least stable gene was invariably $N A C$. In different tissues treated with high temperature, $U B I$ was the best reference gene as determined by the NormFinder algorithm and $18 \mathrm{~S}$ was the best as determined by BestKeeper. However, $18 \mathrm{~S}$ and $U B I$ were the most stable genes according to the results of geNorm and RefFinder. The results obtained by the four methods were consistent in that the most unstable candidate was TUA. Among all samples, $18 S$ was the optimum reference gene, followed by $U B I$. The most unsuitable genes were $N A C$, followed by $T U A$.

It was reported that EFI $\alpha$ in Caragana korshinskii (Yang et al. 2014) and Hordeum brevisubulatum (Zhang et al. 2018), GAPDH in Eleusine coracana (Jatav et al. 2018) and Caragana korshinskii (Yang et al. 2014), and $18 S$ and $T U B$ in Panicum virgatum (Huang et al. 2014) were ideal reference genes under heat stress. However, TUB has exhibited bad performance in Caragana korshinskii (Yang et al. 2014). In this study, $18 S$ isolated from B. striata had the optimal ranking among the seven candidates among different periods of heat stress, followed by TUB. TUA was the least suitable reference gene for Polygonum cuspidatum under different conditions (Wang et al. 2019). In this study, $T U A$ was also suggested to perform badly among different tissues. $N A C$ was found to be an ideal internal control gene in other plant species but was a poor reference gene for $B$. striata.

Validation of the two most stable and unstable candidates were conducted using the target gene BsrbcL, which encodes a constituent of RuBisCO, an important enzyme for plant photosynthesis. These results demonstrate that $18 S$ and $U B I$ are appropriate for transcript normalization in $B$. striata under high temperature stress. 
Moreover, the most suitable reference genes were able to detect a slight decrease in $B s r b c L$. These results demonstrate that reliable reference genes for qRT-PCR analysis were vital for this species and that using inappropriate genes as calibrators may lead to incorrect expression analysis of target genes.

\section{Conclusion}

$18 S$ and $T U B$ were the best reference genes for relative expression analysis of target genes in leaves from Bletilla striata among different periods under heat stress, $18 \mathrm{~S}$ and $U B I$ were the best reference genes among different tissues. Altogether, $18 S$ and $U B I$ were identified to be the best reference genes for all samples.

\section{Acknowledgements}

This work was supported by Aid program for Science and Technology Innovative Research Team of Zhengzhou Normal University, and Science and Technology Project of Henan Province (No. 182102110369).

\section{Author Contributions}

Fang Liang carried out the qRT-PCR and prepared the writing-original draft. Suhua Jiang and Ping'an Hao carried out cloning of the seven genes. Yan Zhang and Shenping $\mathrm{Xu}$ analyzed the data. Suyan Niu and Shiming Han modified the draft and editing. XiuyunYuan and Bo Cui presided over the research.

\section{References}

Andersen CL, JL Jensen, TF Ørntoft (2004). Normalization of real-time quantitative reverse transcription-PCR data: A model-based variance estimation approach to identify genes suited for normalization, applied to bladder and colon cancer data sets. Cancer Res 64:5245-5250

Argyropoulos D, C Psallida, CG Spyropoulos (2006). Generic normalization method for real-time PCR Application for the analysis of the mannanase gene expressed in germinating tomato seed. FEBS J 273:770-777

Bustin SA, V Benes, T Nolan, MW Pfaffl (2005). Quantitative real-time RT-PCR-a perspective. J Mol Endocrinol 34:597-601

Cao LY, XX Li, D Wang, HF Sun, JP Gao (2017). Validation of reliable reference genes for accurate normalization in RT-qPCR analysis of Codonopsis pilosula. Chin Herbal Med 9:226-235

Cheng T, F Zhu, J Sheng, L Zhao, F Zhou, Z Hu, Y Diao, S Jin (2019). Selection of suitable reference genes for quantitive real-time PCR normalization in Miscanthus lutarioriparia. Mol Biol Rep 46:4545-4553

Derveaux S, J Vandesompele, J Hellemans (2010). How to do successful gene expression analysis using real-time PCR. Methods 50:227-230

Diao H, X Li, J Chen, Y Luo, X Chen, L Dong, C Wang, C Zhang, J Zhang (2008). Bletilla striata polysaccharide stimulates inducible nitric oxide synthase and proinflammatory cytokine expression in macrophages. J Biosci Bioeng 105:85-89

Dudziak K, M Sozoniuk, H Szczerba, A Kuzdraliński, K Kowalczyk, A Börner, M Nowak (2020). Identification of stable reference genes for qPCR studies in common wheat (Triticum aestivum L.) seedlings under short-term drought stress. Plant Methods 16; Article 58
Guo JJ, BL Dai, NP Chen, LX Jin, FS Jiang, ZS Ding, CD Qian (2016). The anti-Staphylococcus aureusactivity of the phenanthrene fraction from fibrous roots of Bletilla striata. BMC Complem Altern M 16; Article 491

Gutierrez L, M Mauiat, J Polloux, C Bellini, O Van Wuytswinkel (2008). Towards a systematic validation of references in real-time RT-PCR. Plant Cell 20:1734-1735

Huang L, H Yan, X Jiang, X Zhang, Y Zhang, X Huang, Y Zhang, J Miao, B Xu, T Frazier, B Zhao (2014). Evaluation of candidate reference genes for normalization of quantitative RT-PCR in switchgrass under various abiotic stress conditions. Bioenerg Res 7:1201-1211

Huggett J, K Dheda, S Bustin, A Zumla (2005). Real-time RT-PCR normalization: Strategies and considerations. Genes Immun 6:279-284

Hung HY, TS Wu (2016). Recent progress on the traditional Chinese medicines that regulate the blood. J Food Drug Anal 24:221-238

Jatav PK, A Sharma, DK Dahiya, A Khan, A Agarwal, SL Kothari, S Kachhwaha (2018). Identification of suitable internal control genes for transcriptional studies in Eleusine coracana under different abiotic stress conditions. Physiol Mol Biol Plants 24:793-807

Jiang S, CF Chen, XP Ma, MY Wang, W Wang, Y Xia, N Zhang, MK Wu, WD Pan (2019). Antibacterial stilbenes from the tubers of Bletilla striata. Fitoterapia 138; Article 104350

Kiarash JG, H Dayton Wilde, F Amirmahani, M Mehdi Moemeni, M Zaboli, M Nazari, S Saeed Moosavi, M Jamalvandi (2018). Selection and validation of reference genes for normalization of qRT-PCR gene expression in wheat (Triticum durum L.) under drought and salt stresses. J Genet 97:1433-1444

Kumar K, M Muthamilarasan, M Prasad (2013). Reference genes for quantitative real-time PCR analysis in the model plant foxtail millet (Setaria italic L.) subjected to abiotic stress conditions. Plant Cell Tiss Org Cult 115:13-22

Lee HJ, MG Kim, S Lee, KY Leem (2013). Effects of Bletillae Rhizoma on the elastase, collagenase, and tyrosinase activities and the procollagen synthesis in Hs68 human fibroblasts. Kor J Hernol 28:9-14

Li Q, K Li, SS Huang, HL Zhang, YP Diao (2014). Optimization of extraction process and antibacterial activity of Bletilla striata polysaccharides. Asian J Chem 26:3574-3580

Liao Z, R Zeng, L Hu, KG Maffucci, Y Qu (2019). Polysaccharides from tubers of Bletilla striata: Physicochemical characterization, formulation of buccoadhesive wafers and preliminary study on treating oral ulcer. Intl J Biol Macromol 122:1035-1045

Lin F, L Jiang, Y Liu, Y Lv, HDai, H Zhao (2014). Genome-wide identification of housekeeping genes in maize. Plant Mol Biol 86:543-554

Luo Y, H Diao, S Xia, L Dong, J Chen, J Zhang (2010). A physiologically active polysaccharide hydrogel promotes wound healing. J Biomed Mater Res 94:193-204

Pfaffl MW, A Tichopad, C Prgomet, TP Neuvians (2004). Determination of stable housekeeping genes, differentially regulated target genes and sample integrity: BestKeeper-Excel-based tool using pair-wise correlations. Biotechnol Lett 26:509-515

Qi J, S Yu, F Zhang, X Shen, X Zhao, Y Yu, D Zhang (2010). Reference gene selection for real-time quantitative polymerase chain reaction of mRNA transcript levels in Chinese cabbage (Brassica rapa L. ssp. pekinensis). Plant Mol Biol Rep 28:597-604

Qu Y, C Li, C Zhang, R Zeng, C Fu (2016). Optimization of infraredassisted extraction of Bletilla striata polysaccharides based on response surface methodology and their antioxidant activities. Carbohyd Polym 148:345-353

Shi Y, B Zhang, Y Lu, C Qian, Y Feng, L Fang, Z Ding, D Cheng (2017). Antiviral activity of phenanthrenes from the medicinal plant Bletilla striata against influenza A virus. BMC Complem Altern M 17; Article 273

Shukla P, RA Reddy, KM Ponnuvel, GK Rohela, AA Shabnam, MK Ghosh, RK Mishra (2019). Selection of suitable reference genes for quantitative real-time PCR gene expression analysis in Mulberry (Morus alba L.) under different abiotic stresses. Mol Biol Rep 46:1809-1817

Udvardi MK, T Czechowski, RW Scheible (2008). Eleven golden rules of quantitative RT-PCR. Plant Cell 20:1736-1737 
Vandesompele J, K De Preter, F Pattyn, B Poppe, N Van Roy, A De Paepe, F Speleman (2002). Accurate normalization of real-time quantitative RT-PCR data by geometric averaging of multiple internal control genes. Genome Biol 3; Article RESEARCH0034

Wang X, Z Wu, W Bao, H Hu, M Chen, T Chai, H Wang (2019). Identification and evaluation of reference genes for quantitative realtime PCR analysis in Polygonum cuspidatum based on transcriptome data. BMC Plant Biol 19; Article 498

Wang Y,DLiu, SChen, Y Wang, H Jiang, H Yin (2014). A new glucomannan from Bletilla striata: Structural and anti-fibrosis effects. Fitoterapia 92:72-78

Wu J, J Zhang, Y Pan, H Huang, X Lou, Z Tong (2017). Identification and evaluation of reference genes for normalization in quantitative realtime PCR analysis in the premodel tree Betula luminifera. J For Res 28:273-282

Xie F, P Xiao, D Chen, L Xu, B Zhang (2012). miRDeepFinder: A miRNA analysis tool for deep sequencing of plant small RNAs. Plant Mol Biol 80:75-84

Yang M, S Wu, W You, A Jaisi, Y Xiao (2019). Selection of reference genes for expression analysis in Chinese medicinal herb Huperzia serrata. Front Pharmacol 10; Article 14

Yang Q, J Yin, G Li, L Qi, F Yang, R Wang, G Li (2014). Reference gene selection for qRT-PCR in Caragana korshinskii Kom. under different stress conditions. Mol Biol Rep 41:2325-2334
Yuan XY, SH Jiang, MF Wang, J Ma, XY Zhang, B Cui (2014). Evaluation of internal control for gene expression in Phalaenopsis by quantitative real-time PCR. Appl Biochem Biotechnol 173:1431-45

Zhan X, L Jia, Y Niu, H Qi, X Chen, Q Zhang, J Zhang, Y Wang, L Dong, C Wang (2014). Targeted depletion of tumour-associated macrophages by an alendronate-glucomannan conjugate for cancer immunotherapy. Biomaterials 35:10046-10057

Zhang C, R Zeng, Z Liao, C Fu, H Luo, H Yang, Y Qu (2017). Bletilla striata micron particles function as a hemostatic agent by promoting rapid blood aggregation. Evidence-Based Compl Altern 2017; Article 5820405

Zhang L, Q Zhang, Y Jiang, Y Li, H Zhang, R Li (2018). Reference genes identification for normalization of qPCR under multiple stresses in Hordeum brevisubulatum. Plant Meth 14; Article 110

Zhang M, Q Shao, E Xu, Z Wang, Z Wang, L Yin (2019). Bletilla striata: A review of seedling propagation and cultivation modes. Physiol Mol Biol Plants 25:601-609

Zhuang Y, L Wang, C Liu, H Wang, Y Xu, Y Zhou, S Gu, H Yang, W Xu (2019). A novel fiber from Bletilla striata tuber: Physical properties and application. Cellulose 26:5201-5210

Zhong M, X Yang, Y Hu, L Huang, Y Peng, Z Li, Q Liu, X Wang, X Zhang, G Nie (2020). Identification of candidate reference genes for quantitative RT-PCR in Miscanthus sinensis subjected to various abiotic stresses. Mol Biol Rep 47:2913-2927 